\title{
Large cell anaplastic lymphoma associated with breast implant: a rare case report presentation and discussion of possible management
}

\author{
Domenico Tripodi ${ }^{1 \wedge}$, Maria Ida Amabile ${ }^{1}$, Marzia Varanese ${ }^{1}$, Vito D’Andrea ${ }^{1}$, Salvatore Sorrenti ${ }^{1}$, \\ Claudio Cannistrà ${ }^{2} \wedge$
}

${ }^{1}$ Department of Surgical Sciences, Sapienza University of Rome, Rome, Italy; ${ }^{2}$ Centre Hospitalier Universitaire Bichat Claude-Bernard, Paris, France Correspondence to: Domenico Tripodi. Dipartimento di Scienze Chirurgiche, Università degli Studi di Roma "La Sapienza", Viale Regina Elena 324, CAP 00161 Roma, Italy. Email: domenico.tripodi@uniroma1.it.

\begin{abstract}
Anaplastic large cell lymphomas (ALCL) are a rare type of primary breast lymphoma. The association between breast implants and ALCL was first described in 1997. Breast implant associated (BIA)ALCL arises from the inflammatory $\mathrm{T}$ cells surrounding the fibrous capsule, and most tumors are in situ. Here we present the case of a 60 -year-old woman with ALCL following bilateral silicone breast prosthesis implantation for aesthetic reason. The patient presented at our observation 7 years following the first surgery reporting a sport trauma in the right thoracic region with breast enlargement and tenderness, complaining breast pain at the palpation of the right breast. Imaging study showed a right fluid collection surrounding the affected breast implant. For this reason, the patient underwent bilateral complete capsulectomy (surgical specimen histologically analyzed and resulted negative for ALCL) and implantation of new breast silicone prosthesis. In 10 months, a progressive relapse of the symptoms with a right peri-implant fluid collection restauration was documented and bilateral surgical removal of breast prostheses with right peri-implant capsular biopsy were performed. The histological analysis of the peri-implant capsule laid for ALCL cellules and a new radical surgical excision of bilateral periprothesic capsule was performed followed by adjuvant radiotherapy for a skin relapse of the disease with a subsequent complete remission. No further relapse was observed afterwards. This clinical case raised a series of diagnostic and therapeutic issues highlighting similarities with previous BIA-ALCL reports, but also some peculiarities that deserve to be further investigated and described in order to make an early recognition of the disease for the proper management and treatment of the patient. Due to the rarity of these tumors, rapidly diagnosing a BIA-ALCL case, not neglecting other associated symptoms or signs, is essential to ensure timely and safe treatment.
\end{abstract}

Keywords: Anaplastic large cell lymphoma; case report; breast implant; total capsulectomy; breast cancer

Submitted Dec 02, 2020. Accepted for publication Mar 19, 2021.

doi: $10.21037 / g s-20-853$

View this article at: https://dx.doi.org/10.21037/gs-20-853

\section{Introduction}

Non-Hodgkin's lymphoma (NHL) is a heterogeneous group of hematological malignancies that rarely involve the breast as a primary or secondary tumor. T-cell lymphoma is rare, accounting for only $12 \%$ to $15 \%$ of all NHL in Western countries. Primary NHL of the breast represents less than $1 \%$ of all breast tumors and is predominantly of $\mathrm{B}$ origin (1). Less than $10 \%$ of NHLs derive from mature T-cells, CD30+ $(1,2)$. Large cell anaplastic lymphomas (ALCL) are a rare subtype of NHL cells representing only $3 \%$ of all adult NHL and $6 \%$ of breast NHL (3). ALCL subtypes can be

^ ORCID of Domenico Tripodi: 0000-0002-4190-8168; Vito D’Andrea: 0000-0003-0427-6648; Claudio Cannistrà: 0000-0003-0131-4063. 


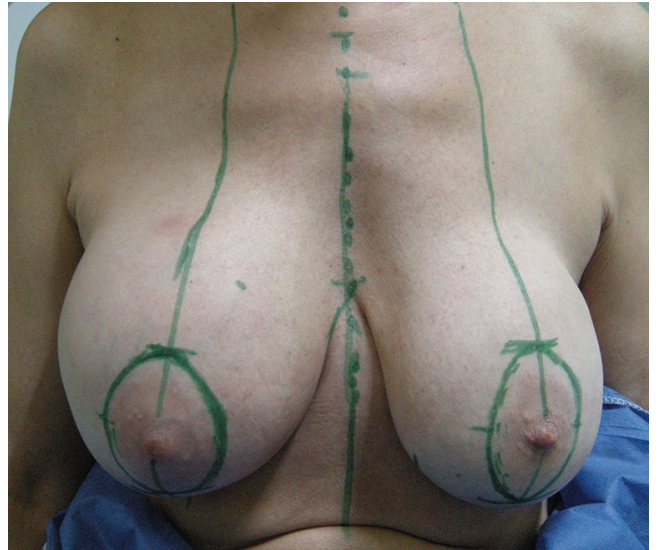

Figure 1 Asymmetry in the right breast prior to surgery.

grouped based on plasma surface kinase (ALK) receptors and also on the primary site of involvement, i.e., primary skin ALCL, breast implant-associated (BIA) ALCL (2). BIA-ALCL is ALK-negative and, similar to ALK-positive ALCL, is generated by eccentric nuclei from a horseshoe shape called "distinctive cells". Unlike other types of ALCL, BIA-ALCL rarely invades beyond the breast. ALK-positive ALCL has been documented mainly in children, while ALKnegative ALCL is more frequent in women between the ages of 40 and 65 and is managed more aggressively (4). All cases of BIA-ALCL are ALK-negative and are derived from the inflammatory T-cells surrounding the periprosthetic fibrous capsule (5). The estimated incidence of BIA-ALCL is 2.03 per 1 million per year with an estimated prevalence of 1 in 30,000 women with breast implants (3). The association between breast implants and ALCL has been discussed in the literature and is extremely rare. The first reported case describing an association between breast implants and ALCL was presented by Keech and Creech in 1997 (6), since then 173 cases have been reported (7). Silicone is the most common material utilized among implantable biomedical devices. In particular, silicone implants are used in surgery for breast augmentation and breast reconstruction. Since their introduction in the 1960s, their safety has been object of debate (1), and the issue of malignant tumors induced by silicone and other filling materials remains controversial (2). The annual incidence of ALCL associated with breast implants is from 0.1 to 0.3 per 100,000 women undergoing breast reconstruction (8) and cases are often underestimated due to the rarity of this cancer. The recognition and acceptance of BIA-ALCL diagnosis by all the physicians involved in the care of patients with breast implants, i.e. surgeons, medical oncologists, and epidemiologists, may lead to be aware of this entity and be able to recognize initial symptoms for the optimal management of this disease (9). We present the following BIA-ALCL case in accordance with the CARE reporting checklist (available at https:// dx.doi.org/10.21037/gs-20-853). This case raised a series of diagnostic and therapeutic issues highlighting similarities with previous BIA-ALCL described reports, but also some peculiarities that deserve to be further investigated and described in order to make an early recognition of the disease, which is essential to ensure timely and safe treatment.

\section{Case presentation}

A 60-year-old woman came to our attention. The patient underwent regular annual breast radiological screening investigations. In the past medical history, we recorded 2 pregnancies, no chronic disease and family history negative for breast and ovarian cancer but positive for a skin lymphoma; 7 years earlier the patient reported bilateral breast augmentation surgery for aesthetic reason, with the insertion of macro-textured silicone retro-muscular breast implants and a regular post-operative course (no infection of the prosthetic pocket or implants' dislocation). Nevertheless, in the last 7 months before to come to our attention she reported a sport trauma of the right thoracic region, with a contusion of the right breast and a hematoma surrounding the right breast implant, complaining breast pain and tenderness at the palpation of the right breast. No fever episodes were reported. At the physical examination, the right breast appeared enlarged, edematous, painful at palpation. The breast ultrasound exam showed a periprosthetic effusion and an ultrasound-guided fineneedle aspiration was performed with bacteriological examination and cytological exam, resulted negative for large anaplastic CD30+ cells. Breast magnetic resonance imaging (MRI) showed no damage of the silicone implants, but a large collection of peri-implant fluid on the right side. The clinical and radiological evaluations of the left breast were within normal ranges. After 4 weeks, the right breast's volume increased again (Figure 1) for persisting of periprosthetic effusion and a second ultrasound-guided fineneedle aspiration was performed, showing at the cytological examination a negative result for lymphoid cells presence. Given this clinic aspect and the radiological imaging, which ruled out prosthetic rupture and/or neoplastic or lymphoproliferative processes, a bilateral total capsulectomy and breast implants replacement were performed. The 


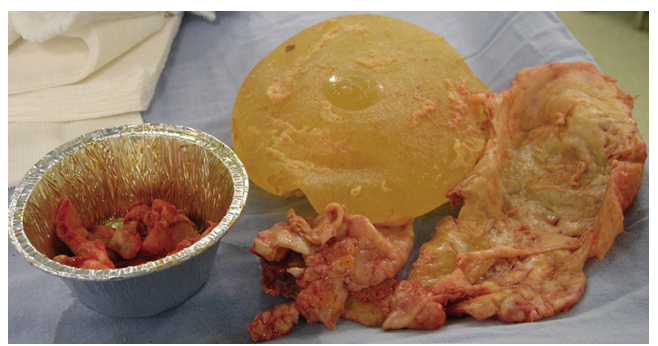

Figure 2 En bloc removal of the breast implant and peri-implant capsule.

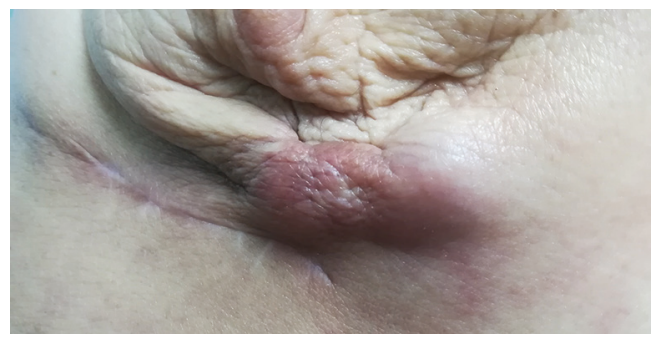

Figure 3 Hyperemic thickening of the right internal lower quadrant after six month surgery.

histological examination of the periprosthetic capsules and the cytological examination of the periprosthetic effusion resulted negative for atypical cells presence as well as the cultural examination was negative for microorganisms' growth (Figure 2). The postoperative course was regular. In 10 months from the last surgery, a progressive increase in the right breast volume was observed again with painful symptoms irradiated also to the ipsilateral axillary region, with hyperemic and edematous skin mainly located in the right lower internal quadrant, and without fever. Moreover, a new periprosthetic fluid effusion was detected at the ultrasound examination and fine-needle aspiration was performed, whose cytological examination did not document any lymphoid cells. Considering the early formation of the periprosthetic fluid collection, the breast implants were permanently removed, and a right periimplant capsular biopsy was performed. At this time, the histological examination of the right periprosthetic capsule revealed the presence of a population of large atypical lymphoid elements, CD30+ and MUM1+ and CD20, CD3, ALK-negative. In addition, the ki67 rate was approximately $80 \%$, confirming the presence of ALCL associated with breast implant (BIA-ALCL), and a new radical surgical excision of bilateral periprothesic capsule was performed.
The TNM classification was pT3 Nx Mx (10). A staging PET-CT scan excluded secondary localizations. In agreement with the hemato-oncologist, a follow-up course was planned. Six months later, a slight hyperemic thickening of the right internal lower quadrant was documented and subjected to diagnostic investigation (Figure 3). For this reason, a breast ultrasound showed a hypoechoic area with irregular and non-homogeneous margin, and the breast MRI showed non-specific hyperintensity in that area. Therefore, a large skin excision of the right internal lower quadrant was performed, whose histological examination confirmed again the presence of lymphoproliferative cells. The patient underwent radiotherapy. The subsequent follow-up investigations resulted negative for disease relapse, as well as the close follow-up clinical exam. No further relapse was observed afterwards. All procedures performed in this study involving human participants were in accordance with the ethical standards of the institutional committee, and with the Helsinki Declaration (as revised in 2013). Written informed consent was obtained from the patient for publication of this case report and any accompanying images.

\section{Discussion and conclusions}

The development of BIA-ALCL is a complex process that may depend on several factors, including the interactions between the host, the immune response, the genetic background of the patient, possible microbial factors, and the surface of the breast implant. BIA-ALCL is supposed to be associated with chronic inflammation (11), which is further supported by scientific observations that chronic inflammation, induced by cytokines or ligands, triggers events that cause genetic instability leading to lymphoma transformation (12). In cases where this disease leads to the formation of only a periprosthetic fluid effusion, it usually follows an indolent clinical course and has an excellent prognosis compared to lymphoproliferative disease with mass formation or to systemic ALK-negative ALCL, which may have more aggressive pathways (13). Patients with BIA-ALCL, undergone total capsulectomy and breast implant remove, have been shown to present a better overall and disease-free survival with respect to patients treated with partial capsulectomy, systemic therapy, or radiotherapy (8). Complete surgical removal of the entire periprosthetic capsule is of utmost importance because the presence of capsule has been associated with disease relapse and progression (14). A potential intraoperative 
pathological study of the margins may allow further tissue removal in case these result not disease-free; however, an intraoperative pathological evaluation is hampered because immediate immunohistochemistry CD30 evaluation is not available. Concerning the timing of reconstructive surgery, there are no standard guidelines. The type and timing of reconstructive surgery are determined by the disease extension, resection capacity, and patient's priorities. We report the case of a patient with BIA-ALCL, who presented 7 years after the implantation of bilateral macrotextured silicone breast implants; even if this patient was successfully treated with two breast implants removal, it is not clear whether the lymphoproliferative disease was related to an initial incomplete resection or to the implant replacement performed during our first surgery treatment. BIA-ALCL is usually a localized disease with an excellent prognosis, although an advanced disease with metastases has also been reported (15). This underlines the importance of early diagnosis and awareness of the extent of the disease, which commonly occurs more as periprosthetic effusion than as a tumor solid mass formation. Skin modifications, capsular contracture, and lymphadenopathy rarely occur (16). In our case, the suspicion of BIA-ALCL was particularly challenging because, although the clinical and radiological imaging were suggestive of BIA-ALCL, the initial cytological analysis of the periprosthetic fluid aspiration did not confirm our suspicion. In addition, our finding of hyperemic skin and the subsequent result of malignant lymphoid cells in the breast skin biopsy performed should lead us to reflect on the best surgical choice to be implemented, consisting in early breast implant removal with a radical capsulectomy (and eventual replacement with non-textured devices) also for those patients who do not have significant capsular pathological modifications even in the absence of prosthetic rupture. However, for any symptom or surgical finding suggesting a lymphoproliferative disease, "enbloc" resection of the peri-prosthetic capsule has to be guaranteed. Interestingly, management of the controlateral breast remains controversial, although several plastic surgeons advise to perform the removal of both implants and capsules because there have been cases of BIA-ALCL identified also in the non-symptomatic breast (9). There is currently no recommended screening for BIA-ALCL early detection. Implant removal is also not recommended in the absence of symptoms. According to the U.S. Food and Drug Administration and the American Society of Plastic Surgeons (5), all women with breast implants should continue routine medical treatment and contact their surgeon in case of changes in breast implants, including pain, swelling, or palpable mass. BIA-ALCL has to be therefore considered in cases of a new peri-implant effusion attributable or not to a trauma that occurs one or more years after the implantation of a textured breast prosthesis. In particular, considering that, except in advanced cases, BIA-ALCL usually has an indolent course with a good prognosis if promptly and properly treated, awareness of the disease among physicians is fundamental to allow an early recognition and to prevent a delay in diagnosis. In this light, an early diagnosis of breast enlargement has to be associated with an examination of the peri-prosthetic liquid and of the peri-prosthetic capsule to be as radical and rapid as possible in removing the altered tissue. The consideration in the patient clinical history of autoimmune diseases or disease of the lymphatic system may lead to foresee the possibility of an increased risk of ALCL, or possibly include the patient in a more periodic and meticulous follow-up. The BIAALCL is treatable if the diagnosis and treatment are rapid and accurate, and prevention can also be based on the identification of family factors predisposing to this disease.

\section{Acknowledgments}

Funding: None.

\section{Footnote}

Reporting Checklist: The authors have completed the CARE reporting checklist. Available at https://dx.doi. org/10.21037/ gs-20-853

Conflicts of Interest: All authors have completed the ICMJE uniform disclosure form (available at https://dx.doi. org/10.21037/gs-20-853). The authors have no conflicts of interest to declare.

Ethical Statement: The authors are accountable for all aspects of the work in ensuring that questions related to the accuracy or integrity of any part of the work are appropriately investigated and resolved. Written informed consent was obtained from the patient for the publication of this clinical case and any accompanying images. All procedures performed in this study involving human participants were in accordance with the ethical standards of the institutional committee, and with the Helsinki Declaration (as revised in 2013). 
Open Access Statement: This is an Open Access article distributed in accordance with the Creative Commons Attribution-NonCommercial-NoDerivs 4.0 International License (CC BY-NC-ND 4.0), which permits the noncommercial replication and distribution of the article with the strict proviso that no changes or edits are made and the original work is properly cited (including links to both the formal publication through the relevant DOI and the license). See: https://creativecommons.org/licenses/by-nc-nd/4.0/.

\section{References}

1. Alexander DD, Mink PJ, Adami HO, et al. The nonHodgkin lymphomas: a review of the epidemiologic literature. Int J Cancer 2007;120 Suppl 12:1-39.

2. Talwalkar SS, Miranda RN, Valbuena JR, et al. Lymphomas involving the breast: a study of 106 cases comparing localized and disseminated neoplasms. Am J Surg Pathol 2008;32:1299-309.

3. Hapgood G, Savage KJ. The biology and management of systemic anaplastic large cell lymphoma. Blood 2015;126:17-25.

4. Drexler HG, Gignac SM, von Wasielewski R, et al. Pathobiology of NPM-ALK and variant fusion genes in anaplastic large cell lymphoma and other lymphomas. Leukemia 2000;14:1533-59.

5. US Food and Drug Administration (2020). Medical devices: questions and answers on anaplastic large cell lymphoma associated with breast implants (BIA-ALCL). Available online: https://www.fda.gov/medical-devices/ implants-and-prosthetics/breast-implants

6. Keech JA Jr, Creech BJ. Anaplastic T-cell lymphoma in proximity to a saline-filled breast implant. Plast Reconstr Surg 1997;100:554-5.

7. Brody GS, Deapen D, Taylor CR, et al. Anaplastic large

Cite this article as: Tripodi D, Amabile MI, Varanese M, D'Andrea V, Sorrenti S, Cannistrà C. Large cell anaplastic lymphoma associated with breast implant: a rare case report presentation and discussion of possible management. Gland Surg 2021;10(6):2076-2080. doi: 10.21037/gs-20-853 cell lymphoma occurring in women with breast implants: analysis of 173 cases. Plast Reconstr Surg. 2015;135:695705. Erratum in: Plast Reconstr Surg 2015;136:426.

8. De Jong D, Vasmel WL, de Boer JP et al. Anaplastic largecell lymphoma in women with breast implants. JAMA 2008;300:2030-5.

9. Leberfinger AN, Behar BJ, Williams NC, et al. Breast Implant-Associated Anaplastic Large Cell Lymphoma: A Systematic Review. JAMA Surg 2017;152:1161-8.

10. NCCN guidelines. Available online: https://www.nccn. org/professionals/physician_gls/default.aspx\#site, accessed on September 2020.

11. Story SK, Schowalter MK, Geskin LJ. Breast implantassociated ALCL: a unique entity in the spectrum of CD30+ lymphoproliferative disorders. Oncologist 2013;18:301-7.

12. Zucca E, Bertoni F, Vannata B, et al. Emerging role of infectious etiologies in the pathogenesis of marginal zone B-cell lymphomas. Clin Cancer Res 2014;20:5207-16.

13. D'Alessandris N, Lucatelli P, Tripodi D, et al. Cytological features of breast implant-associated anaplastic large cell lymphoma in pleural effusion. Diagn Cytopathol 2019;47:1213-7.

14. Clemens MW, Medeiros LJ, Butler CE, et al. Complete Surgical Excision Is Essential for the Management of Patients With Breast Implant-Associated Anaplastic LargeCell Lymphoma. J Clin Oncol 2016;34:160-8. Erratum in: J Clin Oncol 2016;34:888.

15. Quesada AE, Medeiros LJ, Clemens MW, et al. Breast implant-associated anaplastic large cell lymphoma: a review. Mod Pathol 2019;32:166-88.

16. Clemens MW, Jacobsen ED, Horwitz SM. 2019 NCCN Consensus Guidelines on the Diagnosis and Treatment of Breast Implant-Associated Anaplastic Large Cell Lymphoma (BIA-ALCL). Aesthet Surg J 2019;39:S3-S13. 